Int. J. Dev. Biol. 59: 303-311 (2015)

doi: $10.1387 / \mathrm{ijdb} .150198 \mathrm{ml}$

\title{
Selective depolarization of transmembrane potential alters muscle patterning and muscle cell localization in Xenopus laevis embryos
}

\author{
MARIA LOBIKIN', JEAN-FRANÇOIS PARÉ1 ${ }^{1}$ DAVID L. KAPLAN² and MICHAEL LEVIN*,1 \\ ${ }^{1}$ Center for Regenerative and Developmental Biology and Department of Biology, Tufts University and \\ ${ }^{2}$ Department of Biomedical Engineering, Tufts University, Medford, MA, USA
}

\begin{abstract}
The correct anatomical placement and precise determination of specific cell types is required for the establishment of normal embryonic patterning. Understanding these processes is also important for progress in regenerative medicine and cancer biology. Transmembrane voltage gradients across embryonic tissues can mediate cellular communication to regulate the processes of proliferation, migration, and differentiation. Our past work showed that selective depolarization of an endogenous instructor cell population in Xenopus laevis in vivo induced a melanoma-like phenotype in the absence of genetic damage. Here, we use a hypersensitive glycine-gated chloride channel (GlyR) under control of tissue-specific promoters to show that instructor cells resident within muscle are more effective at triggering the metastatic conversion of ectodermal melanocytes than those similar cells within the nervous system. Moreover, depolarization of muscle cells results in aberrant muscle patterning and the appearance of cells expressing muscle markers within the neural tube, which impacts but does not abolish the animals' ability to learn in an associative conditioning assay. Taken together, our data reveal new details of long-range (non-cell-autonomous) reprogramming of cell behavior via alteration of the resting potential of specific embryonic subpopulations.
\end{abstract}

KEY WORDS: development, depolarization, ivermectin, instructor cell, bioelectricity, muscular dystrophy

\section{Introduction}

How cells adopt and maintain their fate is a crucial question in developmental biology. The proper regulation of cell fate and anatomical localization is also an important component of carcinogenesis and metastasis. Alongside the well-studied biochemical signaling cues present in vivo, several types of endogenous bioelectrical signals have also been shown to act as instructive cues for proper tissue patterning and maintenance (Binggeli and Weinstein, 1986, Levin, 2014, McCaig et al., 2005).

Previous work in Xenopus laevis embryos revealed the sensitivity of melanocytes (the pigmented cell derivatives of the embryonic stem cell population known as the neural crest) to the resting potential of specific other cells (Blackiston et al., 2011, Morokuma et al., 2008). Artificially depolarizing a widely-distributed cell population expressing the glycine-gated chloride channel (GlyR) in the developing frog embryo results in the neoplastic-like conversion of melanocytes in the absence of genetic damage or carcinogen exposure. The melanocytes acquire properties associated with cancer: they over-proliferate, change their morphology toward a more dendritic shape, and become highly invasive, targeting heart, neural tube, blood vessels, and visceral organs; aberrant vasculogenesis is also observed (Blackiston et al., 2011, Lobikin et al., 2012). The GlyR-expressing cells were termed "instructor" cells, since they proved able to activate melanocyte metastatic conversion at a distance (non-cell-autonomously).

Remarkably, depolarizing even very small regions of developing Xenopus embryos is sufficient to induce an embryo-wide change in melanocyte behavior (Lobikin et al., 2012). However, two major questions were not answered by body-wide or regional depolarization of instructor cells in their endogenous locations. First,

Abbreviations used in this paper: GlyR, glycine-gated chloride channel; $\mathrm{V}$ transmembrane resting potential.

*Address correspondence to: Dr. Michael Levin. Department of Biology, Tufts University, 200 Boston Avenue, Suite 4600, Medford, MA 02155, USA. Tel: +1-(617)-627-6161. Fax: +1-(617)-627-6121. E-mail: michael.levin@tufts.edu

Supplementary Material (two figures) for this paper is available at: http://dx.doi.org/10.1387/ijdb.150198ml

Accepted: 12 May 2015.

ISSN: Online 1696-3547, Print 0214-6282 
given that the instructor cells are present throughout the embryo but enriched in neural regions such as the ventral neural tube, might one specific sub-population be responsible for the effects we observed? Second, aside from the clearly visible melanocyte phenotype, might additional phenotypes have gone undetected, if they affected non-pigmented cells?

Here, we used tissue-specific promoters to investigate the effects of neural- or muscle-specific instructor cell activation, for the first time addressing individual subsets of this fascinating cell population that is ubiquitous in tissues. In order to depolarize different embryonic lineages, we misexpressed the GlyR channel exclusively in muscle or neural cells. To avoid triggering native instructor cells in other regions of the embryo, we used a hyper-sensitive mutant GlyR which would afford selective targeting of muscle or neural lineages and very low doses of Ivermectin (the GlyR channel opener), which would not depolarize endogenous instructor cells. Depolarizing either muscle or neural-specific instructor cells was sufficient to produce the embryo-wide conversion of melanocyte behavior. Surprisingly, the muscle lineage was more efficient at doing so than the neural lineage. Depolarization resulted in abnormal development of skeletal muscle, and also had the remarkable effect of changing the localization of affected cells to neural regions; cells positive for muscle markers began to appear in the neural tubes of treated animals. These data demonstrate that (1) depolarization of muscle precursors results in abnormal muscle development in vivo, (2) depolarization of even restricted lineages can result in whole-body hyperpigmentation, and (3) depolarization of muscle-specific cells results in their dramatic relocalization to neural regions.

\section{Results}

\section{Depolarization of instructor cells results in a metastatic-like conversion of melanocytes}

We sought to investigate additional effects of selective depolarization of GlyR-expressing cells besides the previously-reported metastatic conversion of melanocytes (Blackiston et al., 2011). GlyR-expressing cells comprise a sparse, yet widely distributed cell population in the developing tadpole (Blackiston et al., 2011) and can be selectively targeted with the drug ivermectin (Ottesen and Campbell, 1994). The extracellular chloride concentration in the Xenopus extracellular media is lower than that of the cytoplasm; thus, when embryos are treated with $1 \mu \mathrm{M}$ ivermectin, native GlyR-channels are opened and chloride ions will flow down their concentration gradient out of the expressing cell, depolarizing it. This depolarizing treatment results in the remarkable embryo-wide conversion of melanocytes, leading to hyperpigmentation in nearly all embryos. We have previously shown that this depolarizing treatment changes three major characteristics of melanocyte behavior: increased proliferation, a more arborized morphology and invasion into ectopic regions (Blackiston et al., 2011). It should be noted that hyperpigmentation is an all-or-none phenotype, with no partial cases observed.

Importantly, the hyperpigmentation phenotype is due to voltage depolarization, and not due to specific effects of chloride concentration, off-target effects of ivermectin, or possible unknown nonchannel roles of GlyR. To confirm this, we reproduced the same phenotype using sodium flux - a strategy often used to interrogate bioelectric signaling (Adams and Levin, 2013). TPC3 is a newly identified two-pore channel, selective for sodium ions, that has an activity profile similar to that of native $\mathrm{Na}_{v}$ channels when injected into Xenopus oocytes (Cang et al., 2014). Injection of this depolarizing voltage-gated sodium channel, TPC3, into one cell of two-cell stage (NF stage 2) embryos also results in the same embryo-wide hyperpigmentation phenotype in $18 \%$ of embryos (Fig. S1). The incidence of hyperpigmentation phenotype was lower than that produced by Ivermectin, since TPC3 mRNA levels had to be kept very low, to avoid major effects on overall development.

\section{Instructor cell depolarization disrupts muscle patterning}

While the change in melanocyte behavior following depolarization is clearly evident through their black pigmentation, effects on other cell types or transparent tissues could have escaped notice. Elegant work previously revealed that mammalian muscle differentiation relied on the function of the potassium channel Kir2.1 (Hinard et al., 2008, Konig et al., 2004), which suggests

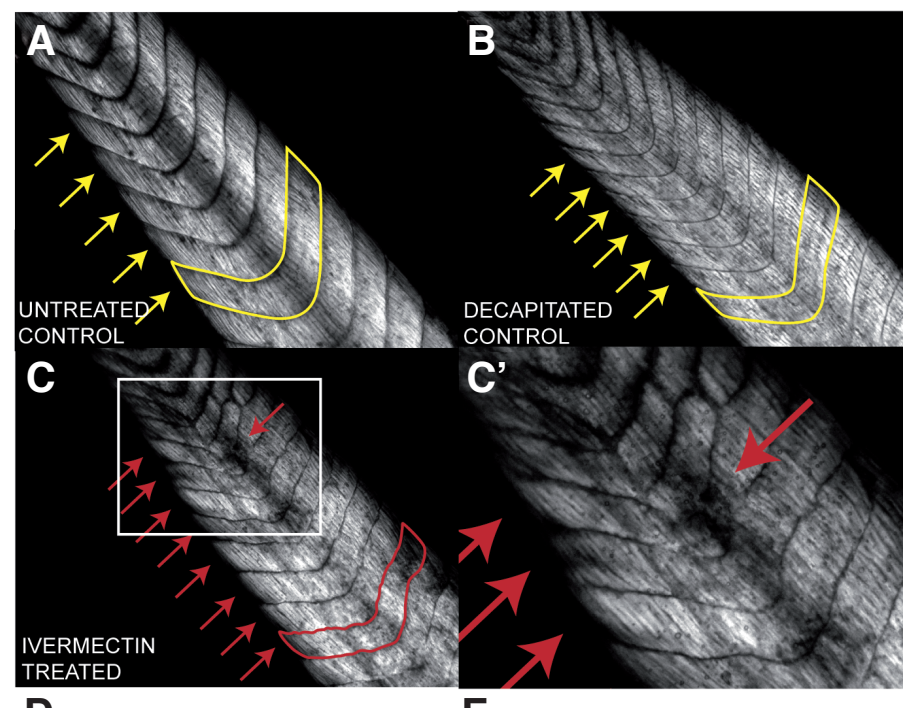

D

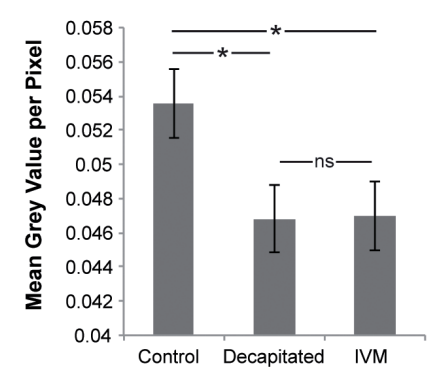

E

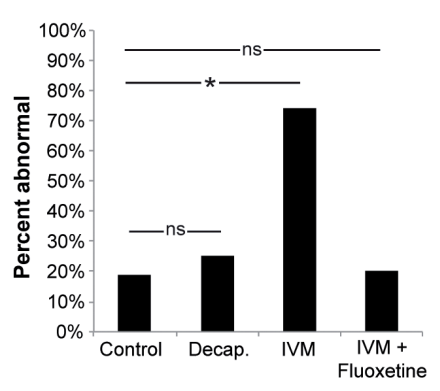

Fig. 1. Depolarization of instructor cells results in abnormal muscle structure independent of immobilization. Birefringence imaging of wildtype (A), decapitated (B) and ivermectin-treated (C, $\left.\mathbf{C}^{\prime}\right)$ tadpole tails reveals disruption of muscle patterning (yellow, red arrows) with depolarizing ivermectin treatment, but not in immobilized tadpoles that had been decapitated at tailbud stage. (D) Quantification of the mean grey value per pixel of area in examined tails reveals a significant decrease in overall collagen density in paralyzed ivermectin-treated and decapitated embryos. (E) Concurrent treatment of $1 \mu \mathrm{M}$ ivermectin with $10 \mu \mathrm{M}$ fluoxetine significantly inhibited ivermectin-induced muscle disorganization (20\% abnormal, $N=20, p<0.01$ compared to ivermectin treatment alone). Error bars denote standard deviation. ${ }^{*}, p<0.01$. 
voltage-dependence of myogenesis in vivo. Thus, we next asked whether skeletal muscle patterning might be affected by instructor cells. Because the pigmentation on the tails of hyperpigmented Xenopus tadpoles obscured visualization of muscle patterning, we examined albino embryos using polarized birefringence imaging.

Birefringence is an intrinsic optical property of the collagen fibers that make up the axial skeletal muscle in the Xenopus tail (van Turnhout et al., 2009). Using polarized light microscopy, tails of albino tadpoles (NF stage 45) that had either been treated with $1 \mu \mathrm{M}$ ivermectin from pre-neurula stage (NF stage 10) onwards, decapitated at NF stage 25/26 (to control for effects of ivermectininduced paralysis on muscle development), or left untreated (controls), were photographed. In normal, untreated tadpoles, the axial muscle of the tail was highly organized - divided into concrete and highly regular segments along the anterior-posterior axis, forming chevron-like structures (Fig. 1A, yellow arrows). Following instructor cell depolarization, the muscle segment peripheries no longer appeared as regular and smooth as in control embryos, and there was a marked loss of muscle collagen signal from some regions in (Fig. $1 \mathrm{C}-\mathrm{D}$ ). This abnormal muscle phenotype was observed in $74 \%(\mathrm{~N}=39)$ of animals examined (Fig. 1E).

Ivermectin treatment has the side effect of paralyzing Xenopus tadpoles. To determine whether the muscle phenotype seen following ivermectin treatment was due to this paralysis, untreated tailbud embryos (NF stage 25/26) were paralyzed via decapitation, and muscle structure was observed at tadpole stages. The collagen organization and chevron pattern of the decapitated tadpoles appeared smooth and organized, as in control embryos (Fig. 1B, yellow arrows), suggesting the muscle phenotype seen following ivermectin treatment is due to depolarization, and not an artifact of the paralysis that this treatment induces. The decapitation, however, did result in a decrease in overall collagen density, as quantified by calculating the brightness (Fig. 1D). We conclude that polarized resting potentials are required for normal muscle development in vivo, and that paralysis induces an overall subtle reduction in collagen level but not the large-scale contiguous defects produced by $\mathrm{V}_{\text {mem }}$ depolarization.

The depolarization-induced hyperpigmentation in Xenopus tadpoles is mediated by serotonin signaling. Inhibition of the voltage-regulated serotonin transporter (SERT) is able to completely prevent depolarization-induced hyperpigmentation (Blackiston et al., 2011). To determine whether serotonin signaling also mediates proper muscle development, embryos were treated with ivermectin together with the SERT inhibitor, fluoxetine, from NF stage 10 onwards, photographed under polarized light at tadpole stages (NF stage 45) and scored for abnormal muscle phenotypes. Exposure to $10 \mu \mathrm{M}$ fluoxetine blocked the ivermectin-induced muscle disorganization, and reduced the percent of abnormal tadpole tails to control levels ( $20 \%$ abnormal, $N=20$, Fig. $1 E$ ). These data suggest that serotonin signaling is important for transducing changes in $\mathrm{V}_{\text {mem }}$ to changes in skeletal muscle patterning.

\section{Tissue-specific instructor cells are sufficient for embryo-wide depolarization}

Hyperpigmentation is an embryo-wide phenotype, and GlyR+ instructor cells are present throughout the body. Since it was not known whether one subset of the instructor cells was responsible for the phenotypes (such as the denser enrichment found within the ventral neural tube), we next sought to determine whether the depolarization of tissue-specific regions is sufficient to induce this embryo-wide change in melanocyte behavior. In order to induce depolarization in specific regions, we took advantage of a Tol2 transposase system and the neural beta tubulin (NBT2) promoter to drive the expression of a hypersensitive GlyR mutant, GlyRA288G (Lynagh and Lynch, 2010), fused to the red fluorescent protein tdTomato, in neural tissue. We confirmed neural-specific expression of GlyR-A288G in embryos that had been injected in
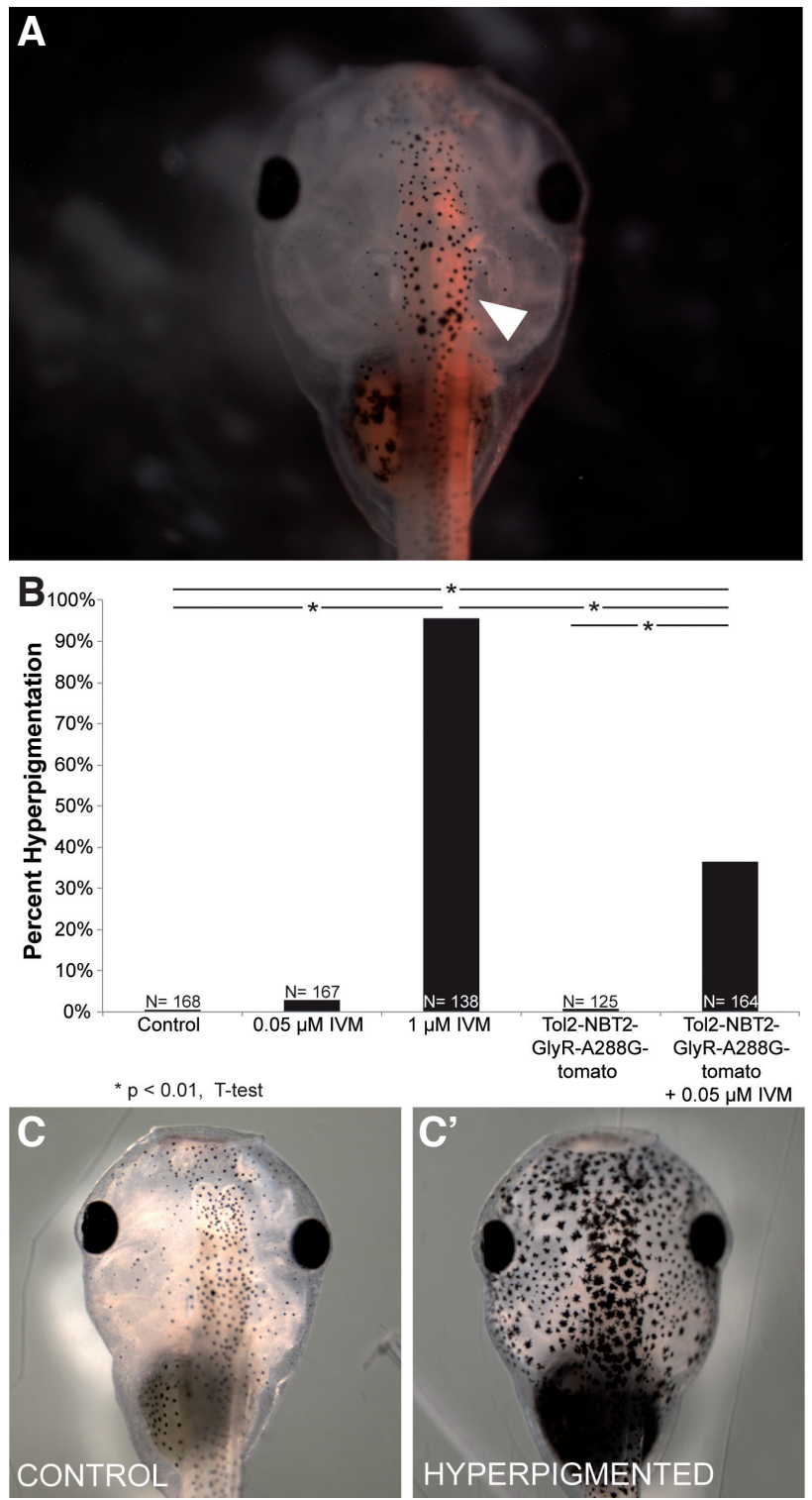

Fig. 2. Driving GlyR-A288G expression in neural tissue is sufficient to induce hyperpigmentation. Embryos were injected withTol2-NBT2-GlyRA288G-tomato (driving the neural-specific expression of a $\mathrm{GlyCl}$ channel with increased sensitivity to ivermectin fused to a tdTomato fluorescent reporter) into 1 cell of 2-cell embryo (NFstage 2). (A) Neural-specific expression was confirmed in NF stage 45 tadpoles (white arrow). (B) Embryos that had been injected with Tol2-NBT2-GlyR-A388G-tom and treated with 0.05 uM ivermectin displayed significant levels $(36.3 \%, N=164, P<<0.01$ in injected \& treated embryos) of hyperpigmentation ( $\left.\mathbf{C}^{\prime}\right)$ compared to control embryos (C) and those that had been injected and not exposed to ivermectin (0.8\% hyperpigmented, $N=125)$. 
one cell at the two-cell stage (NF stage 2) by monitoring fluorescence expression in NF Stage 45 tadpoles (Fig. 2A, white arrow).

By using the GlyR-A288G mutant, we were able to take advantage of its increased sensitivity to ivermectin, and thus open only the introduced (ectopic) channels by using a low dose of ivermectin that does not affect the native GlyR-channels (Fig. 2B). While injection
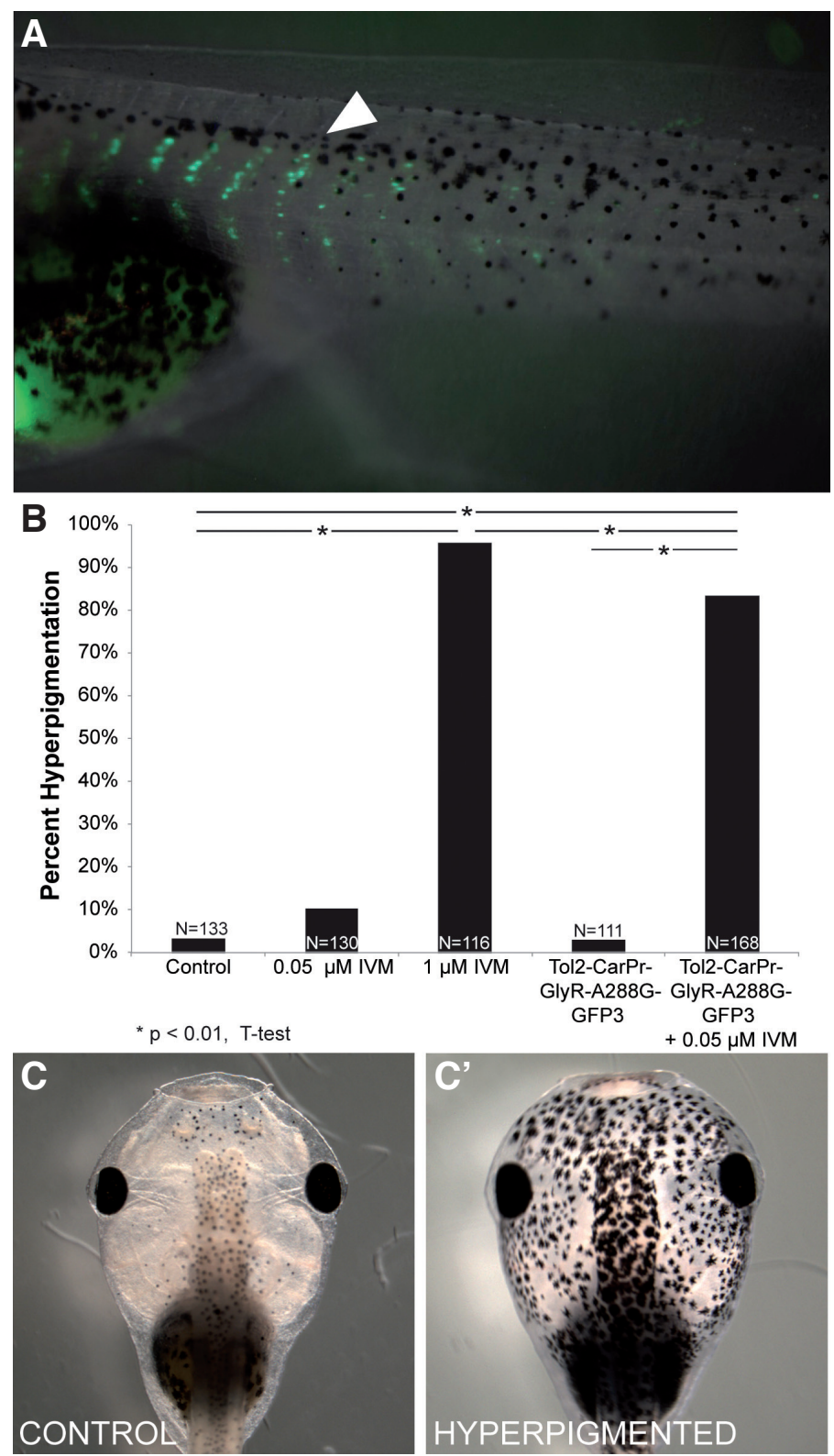

Fig. 3. Driving GlyR-A288G expression in muscle tissue is sufficient to induce hyperpigmentation. Embryos were injected with Tol2-CarPr-GlyRA288G-GFP3 (driving the muscle-specific expression of a GlyR channel with increased sensitivity to ivermectin, fused to a GFP fluorescent reporter) into 1 cell of a 2-cell embryo (NF stage 2). (A) Muscle specific expression of GlyR mutant confirmed in NF stage 45 tadpoles (white arrow). (B) Embryos that had been injected with Tol2-CarPr-GlyR-A288G-GFP3 and treated with a low dose of ivermectin $(0.05 \mu \mathrm{M})$ displayed significant levels $(83.3 \%, N=168, P<<0.01)$ of hyperpigmentation (C") compared to control embryos $\left(\mathbf{C}^{\prime}\right)$ and those that had been injected and not exposed to ivermectin (2.7\% hyperpigmented, $N=111)$. of the Tol2-NBT2pr-GlyR-A288G-tom itself had no effect on overall hyperpigmentation rates, treating injected embryos with $0.05 \mu \mathrm{M}$ ivermectin led to a significant increase in hyperpigmented embryos (Fig. 2B-C, 36.6\% hyperpigmented, $\mathrm{N}=164$, p $<0.01$ ). However, the percentage of hyperpigmented embryos was far less than when embryo-wide instructor cells were targeted (compared to $95.7 \%$ hyperpigmented with $1 \mu \mathrm{M}$ ivermectin treatment of uninjected embryos, Fig. 2B). These data suggest that neural-specific instructor cells are sufficient to induce embryo-wide hyperpigmentation, but not as efficiently as embryo-wide instructor cell depolarization, within the context of the range of expression levels observed in this transgenic system.

Melanocytes themselves are derivatives of a neural embryonic stem cell population known as the neural crest. This cell population forms shortly after neurulation, and then migrates to form a variety of different tissues including neurons, glia, connective tissue, cartilage and melanocytes. Thus, we next wanted to determine whether depolarization of non-neural (and in particular, mesodermal) instructor cells would be sufficient to induce embryo-wide hyperpigmentation. In order to do so, we once again used the Tol2 system, this time driving the expression of the hypersensitive GlyR-A288G mutant in muscle tissue fused to a GFP reporter controlled by the cardiac actin (Car) promoter (Mohun et al., 1986, Mohun et al., 1989). The introduced transgene was first detectable at NF stage 17 (Kroll and Amaya, 1996) and then expressed throughout the life of the animal (data not shown). We confirmed muscle-specific expression in NF stage 45 embryos via fluorescent imaging of tadpole-stage embryos that had been injected with the Tol2-CarPr-GlyR-A288G-GFP3 construct into one cell of the two-cell stage (NF stage 2, Fig. 3A).

While Car promoter-driven GlyR-A288G-GFP3 on its own had no effect on hyperpigmentation rates, treating injected embryos with $0.05 \mu \mathrm{M}$ ivermectin (a dose that does not affect endogenous GlyR channels, Fig. 3B) resulted in a significant percentage of hyperpigmented animals at tadpole stage (Fig. 3B, 83.3\% hyperpigmented, $\mathrm{N}=168, \mathrm{p}<0.01$ ). Surprisingly, depolarizing non-neural instructor cells resulted in more efficient hyperpigmentation than when neural-specific instructor cells were targeted $(83.3 \% \mathrm{vs}$. $36.6 \%$, respectively). Taken together, our data suggest that either muscle- or nerve-specific depolarized cells are able to trigger melanocyte conversion, but that muscle-specific targeting was far more effective (although not quite as effective as depolarizing all of the native instructor cells).

\section{Depolarizing muscle-specific cells results in their partial reprogramming}

While confirming that Tol2-CarPr-GlyR-A288G-GFP3 injections were truly specific to muscle tissue, we looked at the internal localization of the transgenic GFP reporter. Embryos that had been injected with either Tol2-CarPr-GFP3 (as a control) or with Tol2CarPr-GlyR-A288G-GFP3 into one cell at the two-cell stage (NF stage 2) and later treated with ivermectin from NF stage 10 onwards were fixed, sectioned, and processed for immunohistochemistry at tailbud stages (NF stage 28/29) with an anti-GFP antibody. In the control, Tol2-CarPr-GFP-injected embryos, localization of the GFP signal was confined to the somites (Fig. 4A, 4C), as expected. Surprisingly, in embryos in which these muscle-specific cells had been selectively depolarized, Car promoter-driven GFP expression was observed within the neural tube (Fig. 4B) - a location devoid of muscle cells during normal embryogenesis. This improper 
localization of muscle cells was observed in $76 \%$ of embryo sections examined (Fig. 4D). There was no overall difference in the number of $\mathrm{CarPr}^{+}$-cells present between sections of embryos that had been injected with Tol2-CarPr-GFP and embryos injected with Tol2-CarPr-GlyR-A288G-GFP3 and treated with $0.05 \mu \mathrm{M}$ ivermectin (Fig. 4E). This suggests that depolarization does not produce excess GFP+ cells in the neural tube but rather causes these muscle cells to occupy the neural tube at the expense of their presence in their native locations.

To determine whether these improperly localized muscle cells are also positive for other muscle markers, we performed coimmunohistochemistry at tailbud stage (NF stage 28/29) with 2 antibodies: anti-GFP to label injected Car promoter-driven GFP ${ }^{+}$ cells, and anti-12/101 (Kintner and Brockes, 1984), which labels
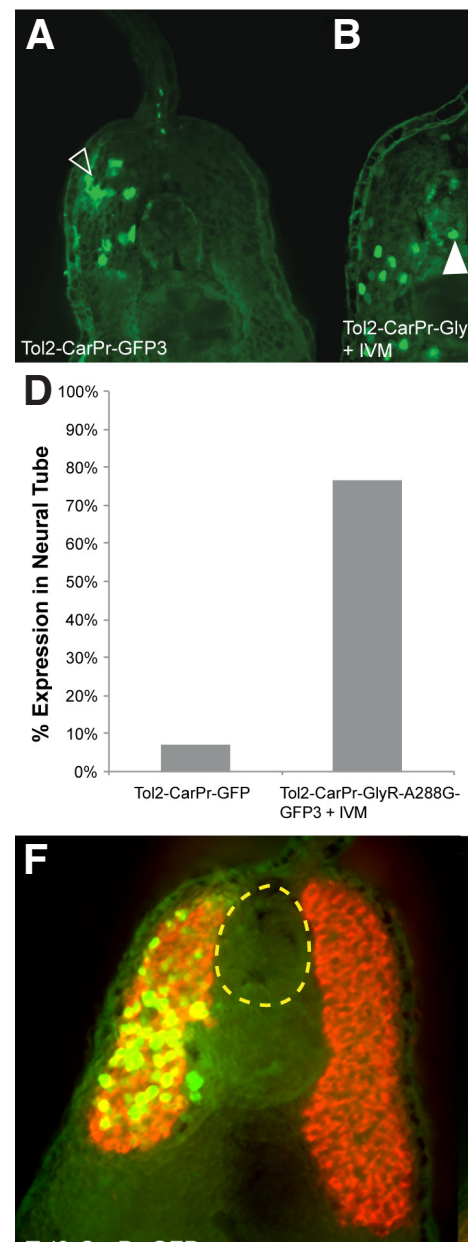

\section{Tol2-CarPr-GFP}

$$
\text { H }
$$

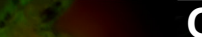

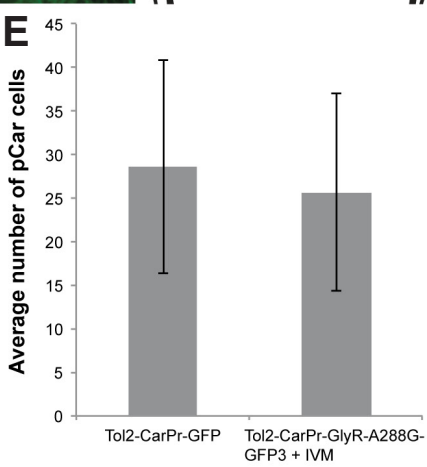

G
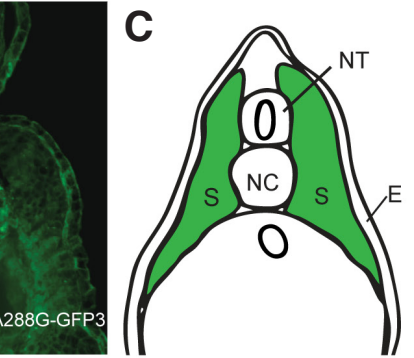

) with Tol2-CarPr-GlyR-A288G-GFP3 butnot treated with ivermectin (Fig. 5B). In sections of embryos that had been injected with Tol2CarPr-GlyR-A288G-GFP3, then treated with $0.05 \mu \mathrm{M}$ ivermectin, while Car promoter driven muscle cells did appear in the neural tube region, they too, did not co-localize with the neural signal, suggesting that these depolarized $\mathrm{CarPr}^{+}$cells that end up in the neural tube are not being completely reprogrammed into neural cells. We were unable to track the anatomical fate of these cells long-term, as the fluorescence of our injected markers disappeared after 1 week. Further studies using transgenic animals, at single-cell and multiple timepoint resolution, are now in progress, to understand the transcriptional and anatomical course of these cells throughout development and subsequent growth.

Fig. 4. Expressing GlyR-A288G and selectively depolarizing musclespecific cells results in abnormal localization. (A) Immunohistochemistry performed on sections of tailbud stage (NF stage 28/29) Xenopus laevis embryos that had been injected with Tol2 construct driving GFP expression in muscle cells using cardiac actin promoter, Car, using GFP-specific antibodies revealed normal muscle specific localization of GFP signal; in somatic tissue, green in (C). (B) Driving hypersensitive GlyR expression (GlyR-A288G) in muscle tissue using Car promoter and subsequent depolarization resulted in GFP signal appearing in neural tissue (white arrow). (D) Abnormal localization of GFP+ cells to the neural tube occurred in $76 \%$ of embryos that had been injected with Tol2-Car-pRGlyR-A288G-GFP3 and treated with $0.05 \mu \mathrm{M}$ ivermectin, but overall number of GFP+ cells was not significantly different between injected and treated embryos and control embryos that had been injected with Tol2-CarPr-GFP3 (E). Co-immunohistochemistry using an anti-GFP antibody to track cells expressing GFP in response to the Car promoter and a 12/101 antibody labeling skeletal muscle confirmed GFP+ cells of Tol2-CarPr-GFP3 injected embryos are localized in somatic muscle tissue (F). Co-immunohistochemistry on embryos that had been injected with Tol2-CarPr-GlyR-A288G-GFP3 and treated with ivermectin displayed either 12/101 signal in the neural tube (G), or Car-regulated GFP signal in the neural tube [white arrow in $\left(\mathbf{H}, \mathbf{H}^{\prime}\right)$ ] but no overlapping signal. Scale bars represent $100 \mu \mathrm{m}$. 
Strikingly, these muscle-promoter driven cells were found to comprise between $2.6 \%$ and $27.3 \%$ (1-6 of $30 \pm 8$ cells) of the cells in a given neural tube (Fig. 5 D,D'). Given the large number of muscle cells colonizing neural regions, we sought to determine whether these embryos display any behavioral changes. Thus, behavior testing was performed using an automated training apparatus that implemented an associative learning paradigm: tadpoles (NF stage 45/46) were to avoid a (moving) red-light section of their petri dish, or receive weak electric shocks (Fig. S2A)(Blackiston and Levin, 2012). Surprisingly, both embryos that had been treated with low doses of ivermectin, and those that had been injected with Tol2-CarPr-GlyR-A288G-GFP3 (at NF stage 2) and then treated with low doses of ivermectin, were able to learn to avoid the red portions of the dish (Fig. S2B). However, embryos that had been both injected with the hypersensitive GlyR channel driven by the CarPr promoter did not learn as effectively as controls. After two training sessions, the uninjected animals displayed a significant aversion to red light, while injected and treated embryos displayed the induced significant aversion to red light following three training sessions (Fig. S2B). No significant differences were observed in average speed or average time being punished during training (Fig. S2 C,D).

Taken together, our data show that depolarizing muscle-specific cells results in their improper colonization of neural regions of the developing Xenopus embryo. Surprisingly, these cells express only one muscle marker at a time (the mature skeletal muscle marker, $12 / 101$, or the pCAR marker), and do not express acetylated alpha tubulin as do real neural tissues. These data suggest that these migrating cells may be partially reprogrammed into an intermediate cell type, or alternatively, that depolarization results in the migration of a non-fully differentiated cell that has already committed to the muscle lineage. Despite the replacement of considerable proportions of neural cells with muscle, learning ability remains (although the speed of learning is decreased).

\section{Discussion}

Melanocyte behavior can be affected by the non-cell-autonomous depolarization of a population of GlyR-expressing instructor cells (Blackiston et al., 2011). In addition to changes in melanocyte behavior and vasculature structure (Lobikin et al., 2012), here we demonstrate that GlyR-mediated depolarization also disrupts normal muscle development (Fig. 1). There is both a loss of density in collagen fibers characteristic of muscular dystrophy and a disorganization of tail muscle patterning. This abnormal muscle phenotype can be rescued by treatment with the specific inhibitor of the serotonin transporter, fluoxetine (Fig. 1E), suggesting that serotonin can prevent the development of the depolarization-induced dystrophic-like muscle phenotype. Serotonin has been previously implicated with instructive signaling downstream of changes in $\mathrm{V}_{\text {mem }}$, mediating changes in melanocyte behavior (Blackiston et al., 2011) as well as in left-right patterning (Fukumoto et al., 2005, Levin, 2005). These data also corroborate a recent finding that fluoxetine treatment can prevent dystrophy in a zebrafish model of Duchenne muscular dystrophy, possibly acting on skeletal muscle to enhance membrane integrity (Waugh et al., 2014). Taken together, these data demonstrate an important role for serotonin in skeletal muscle and suggest a potential therapeutic benefit of serotonin modulation for treating muscular dystrophy.

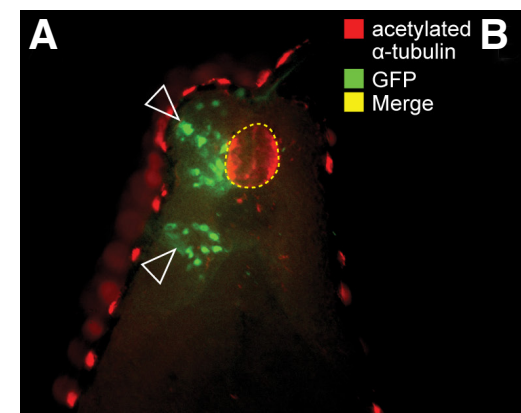

Tol2-CarPr-GFP3

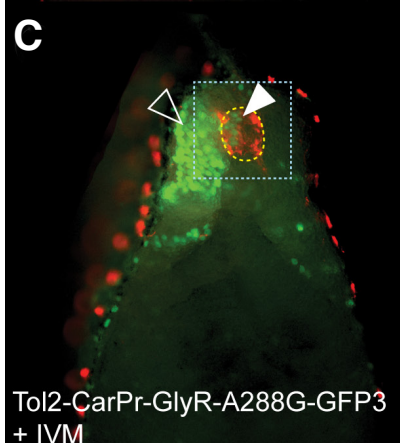

+ IVM

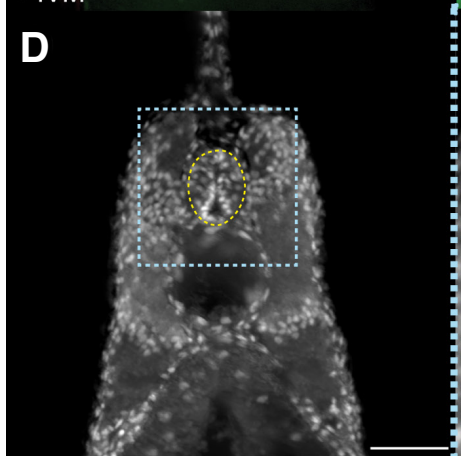

Fig. 5. Mis-localized Car promoter-driven GFP+ cells in neural tube are not completely neural. (A) Double labeling co-immunohistochemistry was performed on sections of tailbud stage (NF stage 28/29) Xenopus embryos that had been injected with Tol2-CarPr-GFP3 using an anti-GFP antibody to track cells that have been activated by the cardiac actin promoter alongside an anti-acetylated $\alpha$-tubulin, labeling neural tissue. This revealed normal expression of GFP in the somatic tissue. (B) Co-immunos on sections of embryos that had been injected with a Tol2-CarPR construct driving expression of hypersensitive GlyR-A288G mutant also revealed normal localization of injected construct. (C) Embryos injected with Tol2CarPr-GlyR-A288G-GFP3 and treated with $0.05 \mu \mathrm{M}$ ivermectin displayed abnormal neural localization (white arrow), however, the GFP+ cells in the neural tube did not co-localize with acetylated $\alpha$-tubulin cells; white arrows in $\left(\mathbf{C}^{\prime}\right)$. (D, $\left.\mathbf{D}^{\prime}\right)$ Hoechst nuclear staining of sections.

Existing data have shown that depolarization of even a few cells, a long distance away from the melanocytes is sufficient to radically change their behavior leading to a metastatic-like phenotype through serotonergic signaling (Lobikin et al., 2012, Morokuma et al., 2008). However, the question as to which subpopulations of cells have the potential to transform these stem cell derivatives remained heretofore unanswered. Here, we demonstrate that these instructor cells do not need to be of a particular cell lineage in order to be able to induce embryo-wide hyperpigmentation: the 
depolarization of either muscle and neural instructor cells is sufficient (Fig. 2B, 3B). The depolarization of muscle-specific cells may be more potent in producing the change in melanocyte phenotype than is the depolarization of cells of a neural lineage (Fig. 2B, 3B). This is especially interesting since it is known that the expression pattern of GlyR-expressing cells in Xenopus is denser in the ventral marginal zone of the neural tube than the punctate distribution of these cells throughout the entire embryo (Blackiston et al., 2011), and it is that region which initially was thought to be responsible for the hyperpigmentation induced by embryos'exposure to ivermectin. These differences in hyperpigmentation phenotype penetrance could formally be due to differences in expression levels, as we could not verify that in every embryo injected with the NBT-promoter driven there was strong expression across the entire central nervous system. However, in our hands, the overall expression levels from each of these promoters were quite comparable. Another possibility, that the difference in number of cells expressing the neural vs. muscle promoter is what accounts for the different penetrance of phenotype, is probably unlikely since we have previously shown that very few depolarized instructor cells are needed to trigger the animal-wide hyperpigmentation phenotype (Lobikin et al., 2012).

Importantly, the depolarized muscle cells that induce hyperpigmentation are themselves being altered while signaling to the melanocytes. Here, we demonstrate for the first time, how driving the specific depolarization of muscle cells during development leads to their partial reprogramming in vivo. While these cells driving the Car promoter are normally found in muscle-specific regions of the developing Xenopus tadpole (Fig. 4C), selective depolarization caused them to take up residence in the neural tube (Fig. 4B, 4H, 5C) - a highly abnormal location for muscle cells. These ectopic cells only express one muscle marker at a time of the pair tested (pCAR and 12/101) (Fig. 4 G,G'), and do not express the neural-specific marker acetylated alpha-tubulin (Fig. 5 C,C'). These ectopic cells also have a modest effect on the ability of tadpoles in learning studies, as it takes embryos subjected muscle-specific depolarization one extra training period to learn to avoid red light (Fig. S2A). While there was no significant difference in the behavior of these tadpoles from control tadpoles that had been treated with low doses of ivermectin alone, it is important to note that there is an increase in variance of recall performance between the injected and treated tadpoles, suggesting that a subset of tadpoles were indeed affected by the presence of ectopic cells in neural regions.

The cells migrating into the neural tube may be partially reprogrammed into an intermediate cell type, or may result from the migration of a non-fully differentiated cell that has already committed to the muscle lineage. The latter alternative suggests that only non-differentiated cells are targeted for depolarization. Neural beta-tubulin is expressed exclusively in differentiated neurons in Xenopus, whereas cardiac actin in the embryo is expressed in the myotomes, which will later become muscles. Therefore, what may be occurring in injected and treated embryos is a change in migration of cells that have committed enough towards muscle lineage that by the time they migrate into the neural tube, they can no longer become neural. These migrating cells are removed from the regular muscular environment and so no longer commit to muscle, while the rest of the cells in the somites carry on down muscular differentiation pathways. This may explain the difference in penetrance of the two Tol2 constructs; one is expressed in a differentiated environment whereas the other is in a precursor cell that is committed to a certain pathway but has not yet terminally differentiated.

The ultimate fate of these misplaced muscle cells remains unknown, as tracking these cells at late stages has proven to be difficult due to progressive disappearance of the GFP protein with developmental time. Future work will seek to determine whether these cells move to the neural tube from an initially normal location or whether existing neural tube cells become more muscle-like. While in vivo cell motility data are not yet available, we favor the former hypothesis because of the unchanged total number of CARPR $^{+}$cells in depolarized embryos.

In normal development, embryonic stem cells gradually progress from a multipotent state, capable of generating all cell types, to a highly restricted state by sequential restriction of differentiation potential (Chambers and Studer, 2011). These differentiation processes have been shown to be subject to manipulation from various signaling cues, such as transcription factor expression (Mong et al., 2014, Weintraub et al., 1989) but have also been shown to be directed by bioelectrical events (Barth and Barth, 1974, Lange et al., 2011, Sundelacruz et al., 2009). While much of this work has been done in vitro, our data suggest that in vivo depolarization can also affect cell fate determination. The depolarization of cells in which gene expression is driven by the cardiac actin promoter (i.e. specific to muscle tissue) results in these cells no longer being confined to the segmented blocks of mesoderm destined to become muscle (i.e. the somites), but rather in the ectodermal region of the neural tube. This suggests that bioelectric state may be an important component of proper germ cell layer specification events that occur during gastrulation in the developing frog embryo.

It is now clear that the misregulation of stem cell fate and division is also an important component of cancer (Morrison and Kimble, 2006). Given its characteristic morphogenetic derangement, cancer can also be thought of as a developmental disorder (Chernet and Levin, 2013, Levin, 2009), and the Xenopus tadpole system is an ideal model for understanding this interplay between normal developmental signals and those as manifest as cancer. Perturbations that induce metastatic-like phenotypes during development may provide great insight into the signals that create cancer stem cells. We see here that in addition to observations of the main properties of metastasis (overproliferation, cell shape change promoting invasion, and ectopic colonization) seen in altered melanocytes, overall muscle structure is also affected (Fig. 1). Interestingly, despite this subtle deficiency, tadpoles with depolarized instructor cells appear to have no gross anatomical defects. However, future work with animals with other fluorescently-labeled cell types or with more detailed marker analysis in sections may reveal additional cell populations that respond to bioelectric changes in instructor cells. Of course, the search for new instructor cells (demarcated by other characteristic ion channels through which they can be selectively depolarized) is another active area of research.

Taken together, these studies reveal how changes in $\mathrm{V}_{\text {mem }}$ can function in the morphogenetic regulation of both melanocytes and muscle cells, relevant not only to developmental patterning but also to cancer. Hopefully, a better understanding of how these bioelectrical signals function together with the well studied molecular and biochemical pathways will provide insights to several branches of biomedicine. 


\section{Materials and Methods}

\section{Animal husbandry}

Xenopus embryos were maintained according to standard protocols (Sive et al., 2000) in 0.1× Modified Marc's Ringers (MMR), pH 7.8. Xenopus embryos were staged according to Nieuwkoop and Faber (Nieuwkoop and Faber, 1994). Transgenic Xenopus laevis embryos were prepared by Gufa Lin using plasmids from Enrique Amaya. Animal handling conducted in accordance with protocol M2014-79.

\section{Microinjection}

Capped, synthetic mRNAs were dissolved in water and injected into embryos at cleavage stages in 3\% Ficoll using standard methods (Sive et al., 2000). mRNA injections were made into specific locations using borosilicate glass needles calibrated to bubble pressures of $50-70 \mathrm{kPa}$ in water, delivering 50-70 ms pulses. After $30 \mathrm{~min}$, embryos were washed and cultured in 0.1X MMR until desired stages. eGFP-TPC3 construct was received from Chunlei Cang (Cang et al., 2014) and subcloned into PCS2 for the synthesis of messenger RNA. Other constructs used included Tol2-CarPr-GFP3, Tol2-CarPr-GlyR-A288G-GFP3 (in which human GlyRA288G (Lobikin et al., 2012, Lynagh and Lynch, 2010) was inserted into Tol2-CarPr-GFP3), Tol2-NBT2-GlyR-A288G-tomto (made by replacing GFP3 in Tol2-NBT2-GFP3 (Pai, 2015) with GlyR-A288G-tomato). Cardiac actin promoter and neural tubulin promoters were the same as those used for transgenic frogs. Tol2 constructs were injected as DNA following the same protocol used for mRNA injections.

\section{Drug exposure}

Stocks of ivermectin (Sigma) were stored at $10 \mathrm{mM}$ concentration in dimethyl sulfoxide (DMSO). Embryos were exposed in $0.1 \mathrm{X}$ MMR from pre-neurulation (NF stage 10) to tadpole stage (NF stage 45) to ivermectin, $1 \mu \mathrm{M}$ or $0.05 \mu \mathrm{M}$, or fluoxetine, $10 \mu \mathrm{M}$.

\section{Immunohistochemistry}

Xenopus embryos were collected and fixed in MEMFA [100 mM 3-(N morpholino) propanesulfonic acid ( $\mathrm{pH} 7.4), 2 \mathrm{mM}$ ethylene glycol tetraacetic acid, $1 \mathrm{mM} \mathrm{MgSO}$, $3.7 \%$ (v/v) formaldehyde] at tailbud stage (NF stage 28/29) for $1 \mathrm{hr}$ (Sive et al., 2000), washed twice in phosphate buffered saline Tween-20 (PBST), embedded into Agarose and sectioned at 200 um using a vibratome (Leica VT1000S) according to previously described protocols (Blackiston et al., 2011). Samples were then blocked with 10\% normal goat serum in PBST for $1 \mathrm{hr}$ at room temperature. Samples were rocked overnight at $4^{\circ} \mathrm{C}$ using the following antibodies: Anti-GFP (AbCam AB290, used at 1:500 dilution), 12/101 (DHSB, concentrate used at 1:100) and Anti-acetylated alpha tubulin (Sigma T7451 used at 1:500 dilution) antibodies were used for immunohistochemistry using previously described protocols (Levin, 2004). Following primary exposure, samples were washed three times in PBST before a 60-min secondary incubation with either AlexaFluor-555 or AlexaFluor-488 conjugated secondary used at 1:1000 diluted in PBST. Following secondary incubation, samples were washed three times for $15 \mathrm{~min}$ in PBST and imaged on an Olympus BX61 microscope.

\section{Birefringence imaging \& analysis}

Birefringence microscopy was performed on an Olympus BX-61 compound microscope with a universal condenser (U-UCD8). The transmitted light DIC slider (U-DICTS) was pulled out and the polarizing filter was rotated such the background appeared darkest. Embryo tails were positioned at a $45^{\circ}$ angle for imaging. Analysis of birefringence images was performed in ImageJ. The mean grey value of each tail section was calculated, and normalized to tail fragment size.

\section{Behavior testing}

Behavioral testing was conducted using an automated training apparatus that has been documented previously (Blackiston et al., 2010, Blackiston and Levin, 2012). The overall structure of the device includes a rectangular array of cells, each with a disposable Petri dish in the field of vision of a machine vision camera (Insight-Micro 1400, Cognex Corporation, Natick, MA, USA). Each Petri dish housed a single animal that can be subjected to a series of individual conditions, with external illumination of each dish provided by red and/or blue LEDs (Osram Semiconductors, Sunnyvale, CA, USA; blue LED, $470 \mathrm{~nm}$, part no. LBW5SM; red LED, $635 \mathrm{~nm}$, part no. LRG6SP) housed in an illumination control module (ICM). In this device, illumination is delivered from above the experimental environment and can vary both by quadrant and intensity, ranging between maximum or off in fifteen even steps. Within each cell is also a set of six iridium oxide-coated titanium electrodes, allowing the delivery of mild to strong electric shocks. All shocks delivered during light-mediated training experiments were 1.2 $\mathrm{mA} \mathrm{AC}$ currents, pulsed for $100 \mathrm{~ms}$, followed by $300 \mathrm{~ms}$ of no shock. Computer-controlled software executed an experiment autonomously, recording the animal's position and speed in the dish, as well as changing lighting conditions or delivering shock as a result of time and/or behavior criteria specified in the training paradigm. All positional data during each trial were written to a log file, which was parsed and analyzed in Excel over discrete time blocks.

\section{Acknowledgements}

We thank Amanda Allen and Erin Switzer for general lab assistance and frog husbandry, Gufa Lin for the CarPr-GFP transgenic frogs (Linetal., 2012), Joan Lemire for molecular biology assistance, Doug Blackiston for behavior testing assistance, and the members of the Levin lab and the bioelectricity community for many useful discussions. We thank Doug Blackiston, Gary McDowell, and Joan Lemire for comments on a draft of the manuscript. We gratefully acknowledge the support of AHA(14IRG18570000), (DARPA \#W911NF-11-2-0054), NIH (AR061988; AR00559I), The G. Harold and Leila Y. Mathers Charitable Foundation and the W.M. Keck Foundation.

\section{References}

ADAMS, D.S. and LEVIN, M. (2013). Endogenous voltage gradients as mediators of cell-cell communication: strategies for investigating bioelectrical signals during pattern formation. Cell Tissue Res. 352: 95-122.

BARTH, L.G. and BARTH, L.J. (1974). Ionic regulation of embryonic induction and cell differentiation in Rana pipiens. Dev. Biol. 39: 1-22.

BINGGELI, R. and WEINSTEIN, R.C. (1986). Membrane potentials and sodium channels: hypotheses for growth regulation and cancer formation based on changes in sodium channels and gap junctions. J. Theor. Biol. 123: 377-401.

BLACKISTON, D., ADAMS, D.S., LEMIRE, J.M., LOBIKIN, M. and LEVIN, M. (2011). Transmembrane potential of GlyCl-expressing instructor cells induces a neoplastic-like conversion of melanocytes via a serotonergic pathway. Disease Mod. Mech. 4: 67-85.

BLACKISTON, D., SHOMRAT, T., NICOLAS, C.L., GRANATA, C. and LEVIN, M. (2010). A second-generation device for automated training and quantitative behavior analyses of molecularly-tractable model organisms. PloS One 5: e14370.

BLACKISTON, D.J. and LEVIN, M. (2012). Aversive training methods in Xenopus laevis: general principles. CSH Protocols 5: (10.1101/pdb.top068338)

CANG, C., ARANDA, K. and REN, D. (2014). Anon-inactivating high-voltage-activated two-pore $\mathrm{Na}(+)$ channel that supports ultra-long action potentials and membrane bistability. Nat Commun 5: 5015.

CHAMBERS, S.M. and STUDER, L. (2011). Cell fate plug and play: direct reprogramming and induced pluripotency. Cell 145: 827-30.

CHERNET, B. and LEVIN, M. (2013). Endogenous Voltage Potentials and the Microenvironment: Bioelectric Signals that Reveal, Induce and Normalize Cancer. J. Clin. Exp. Oncol. Suppl 1. (doi:10.4172/2324-9110.S1-002).

DAS, B., SCHREIBER, A.M., HUANG, H. and BROWN, D.D. (2002). Multiple thyroid hormone-induced muscle growth and death programs during metamorphosis in Xenopus laevis. Proc. Natl. Acad. Sci. USA 99: 12230-12235.

FUKUMOTO, T., BLAKELY, R. and LEVIN, M. (2005). Serotonin transporter function is an early step in left-right patterning in chick and frog embryos. Dev. Neurosci. 27: 349-363. 
HINARD, V., BELIN, D., KONIG, S., BADER, C.R. and BERNHEIM, L. (2008). Initiation of human myoblast differentiation via dephosphorylation of Kir2.1 $\mathrm{K}+$ channels at tyrosine 242. Development 135: 859-867.

KINTNER, C.R. and BROCKES, J.P. (1984). Monoclonal antibodies identify blastemal cells derived from dedifferentiating limb regeneration. Nature 308: 67-69.

KONIG, S., HINARD, V., ARNAUDEAU, S., HOLZER, N., POTTER, G., BADER, C.R. and BERNHEIM, L. (2004). Membrane hyperpolarization triggers myogenin and myocyte enhancer factor-2 expression during human myoblast differentiation. $J$. Biol. Chem. 279: 28187-28196.

KROLL, K.L. and AMAYA, E. (1996). Transgenic Xenopus embryos from sperm nuclear transplantations reveal FGF signaling requirements during gastrulation. Development 122: 3173-3183.

LANGE, C., PRENNINGER, S., KNUCKLES, P., TAYLOR, V., LEVIN, M. and CALEGARI, F. (2011). The $\mathrm{H}(+)$ vacuolar ATPase maintains neural stem cells in the developing mouse cortex. Stem Cells. Dev. 20: 843-850.

LEVIN, M. (2005). Left-right asymmetry in embryonic development: a comprehensive review. Mech. Dev. 122: 3-25.

LEVIN, M. (2009). Errors of geometry: regeneration in a broader perspective. Sem. Cell. Dev. Biol. 20: 643-645.

LEVIN, M. (2014). Molecular bioelectricity: how endogenous voltage potentials contro cell behavior and instruct pattern regulation in vivo. Mol. Biol. Cell 25: 3835-3850.

LIN, G.F., CHEN, Y. and SLACK, J.M.W. (2012). Transgenic Analysis of Signaling Pathways Required for Xenopus Tadpole Spinal Cord and Muscle Regeneration. Anat. Rec. -Adv. Int. Anat. Evol. Biol. 295: 1532-1540.

LOBIKIN, M., CHERNET, B., LOBO, D. and LEVIN, M. (2012). Resting potential, oncogene-induced tumorigenesis, and metastasis: the bioelectric basis of cancer in vivo. Phys. Biol. 9: 065002.

LYNAGH, T. and LYNCH, J.W. (2010). An improved ivermectin-activated chloride channel receptor for inhibiting electrical activity in defined neuronal populations. J. Biol. Chem. 285: 14890-14897.

MCCAIG, C.D., RAJNICEK, A.M., SONG, B. and ZHAO, M. (2005). Controlling cell behavior electrically: current views and future potential. Physiol Rev 85: 943-978.
MOHUN, T.J., GARRETT, N. and GURDON, J.B. (1986). Upstream sequences required for tissue-specific activation of the cardiac actin gene in Xenopus laevis embryos. EMBO J 5: 3185-3193.

MOHUN, T.J., TAYLOR, M.V., GARRETT, N. and GURDON, J.B. (1989). The CArG promoter sequence is necessary for muscle-specific transcription of the cardiac actin gene in Xenopus embryos. EMBO J 8: 1153-1161.

MONG, J., PANMAN, L., ALEKSEENKO, Z., KEE, N., STANTON, L.W., ERICSON J. and PERLMANN, T. (2014). Transcription factor-induced lineage programming of noradrenaline and motor neurons from embryonic stem cells. Stem cells 32 609-622

MOROKUMA, J., BLACKISTON, D., ADAMS, D.S., SEEBOHM, G., TRIMMER B. and LEVIN, M. (2008). Modulation of potassium channel function confers a hyperproliferative invasive phenotype on embryonic stem cells. Proc. Natl. Acad. Sci. USA105: 16608-16613.

MORRISON, S.J. and KIMBLE, J. (2006). Asymmetric and symmetric stem-cell divisions in development and cancer. Nature 441: 1068-1074.

OTTESEN, E.A. and CAMPBELL, W.C. (1994). Ivermectin in human medicine. The J. Antimicrob. Chemother. 34: 195-203.

PAI, V., LEMIRE, J. M., PARE, J-F., LIN, G., CHEN, Y., AND LEVIN, M.,. (2015). Endogenous gradients of resting potential instructively pattern embryonic neural tissue via Notch signaling and regulation of proliferation. J. Neuroscience.

SUNDELACRUZ, S., LEVIN, M. and KAPLAN, D.L. (2009). Role of membrane potentia in the regulation of cell proliferation and differentiation. Stem Cell Rev. 5:231-246.

VANTURNHOUT, M.C., KRANENBARG, S. and VAN LEEUWEN, J.L. (2009). Modeling optical behavior of birefringent biological tissues for evaluation of quantitative polarized light microscopy. J Biomed Opt 14: 054018.

WAUGH, T.A., HORSTICK, E., HUR, J., JACKSON, S.W., DAVIDSON, A.E., LI, X and DOWLING, J.J. (2014). Fluoxetine prevents dystrophic changes in a zebrafish model of Duchenne muscular dystrophy. Hum Mol Genet 23: 4651-4662.

WEINTRAUB, H., TAPSCOTT, S.J., DAVIS, R.L., THAYER, M.J., ADAM, M.A., LASSAR, A.B. and MILLER, A.D. (1989). Activation of Muscle-Specific Genes in Pigment, Nerve, Fat, Liver, and Fibroblast Cell-Lines by Forced Expression of Myod. Proc. Natl. Acad. Sci. USA 86: 5434-5438. 


\section{Further Related Reading, published previously in the Int. J. Dev. Biol.}

Egg activation in physiologically polyspermic newt eggs: involvement of IP3 receptor, PLCY, and microtubules in calcium wave induction

Tomoyo Ueno, Takehiro Ohgami, Yuichirou Harada, Shuichi Ueno and Yasuhiro Iwao

Int. J. Dev. Biol. (2014) 58: 315-323

Visualization, characterization and modulation of calcium signaling during the development of slow muscle cells in intact zebrafish embryos

Chris Y. Cheung, Sarah E. Webb, Donald R. Love and Andrew L. Miller

Int. J. Dev. Biol. (2011) 55: 153-174

Could modifications of signalling pathways activated after ICSI induce a potential risk of epigenetic defects?

Brigitte Ciapa and Christophe Arnoult

Int. J. Dev. Biol. (2011) 55: 143-152

Oscillatory $\mathrm{Ca} 2+$ dynamics and cell cycle resumption at fertilization in mammals: a modelling approach

Geneviève Dupont, Elke Heytens and Luc Leybaert

Int. J. Dev. Biol. (2010) 54: 655-665

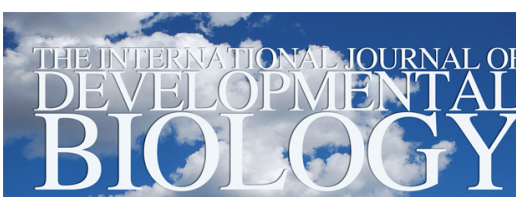

Volume 56 Nos. $10 / 11 / 12$ Special Issue
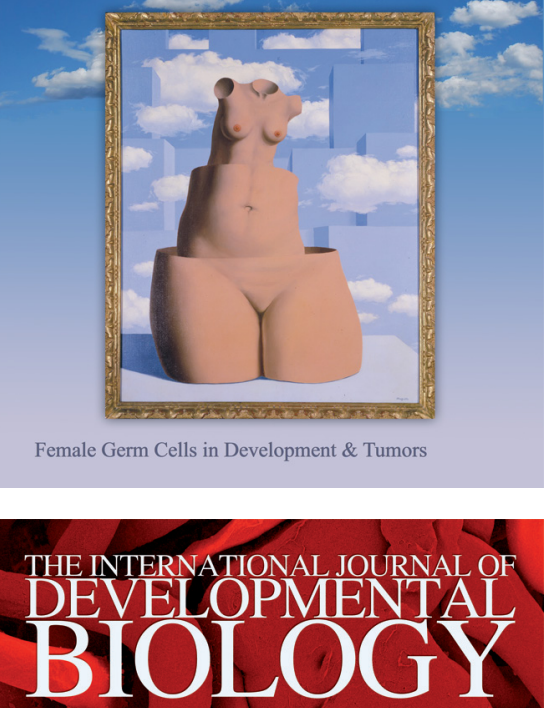

Volume 57 Nos. 2/3/4

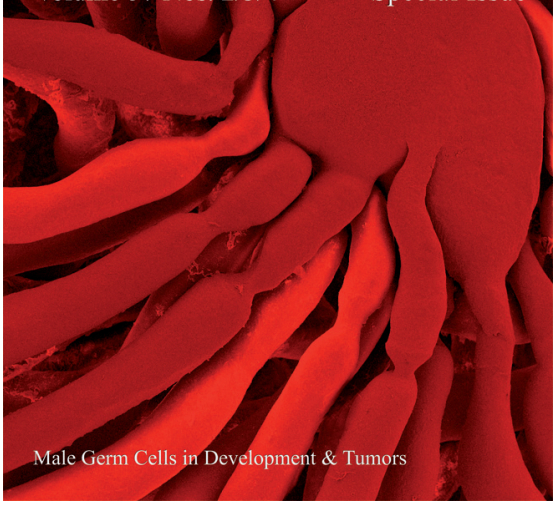

The role of ion fluxes in polarized cell growth and morphogenesis: the pollen tube as an experimental paradigm

Erwan Michard, Filipa Alves and José A. Feijó

Int. J. Dev. Biol. (2009) 53: 1609-1622

Sperm-activating peptides in the regulation of ion fluxes, signal transduction and motility Alberto Darszon, Adán Guerrero, Blanca E. Galindo, Takuya Nishigaki and Christopher D. Wood Int. J. Dev. Biol. (2008) 52: 595-606

The dynamics of calcium oscillations that activate mammalian eggs Karl Swann and Yuansong Yu

Int. J. Dev. Biol. (2008) 52: 585-594

The choice between epidermal and neural fate: a matter of calcium. Marc Moreau and Catherine Leclerc

Int. J. Dev. Biol. (2004) 48: 75-84
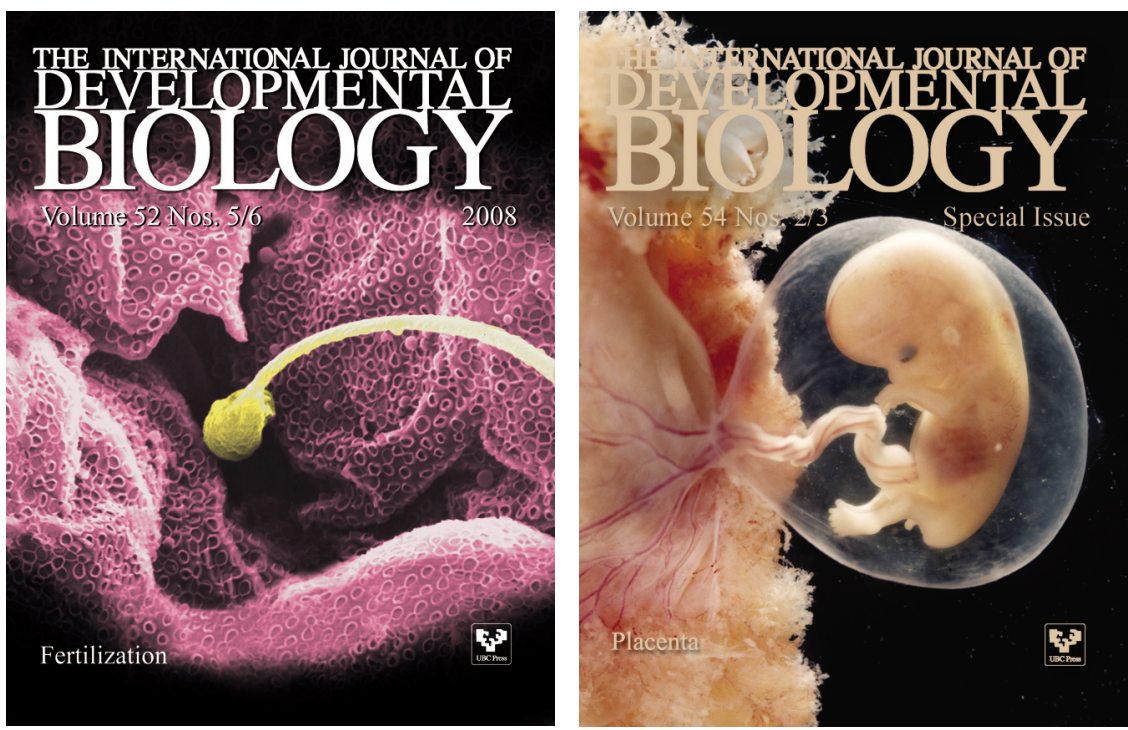